\title{
Quantum Dynamics of Water from Møller-Plesset Perturbation Theory via a Neural Network Potential
}

\author{
Jinggang Lan \\ Department of Chemistry, University of Zurich, Winterthurerstrasse 190, Zurich 8057, Switzerland and \\ Current address: Chaire de Simulation à l'Echelle Atomique (CSEA), \\ Ecole Polytechnique Fédérale de Lausanne (EPFL), CH-1015 Lausanne, Switzerland; \\ David M. Wilkins \\ Atomistic Simulation Centre, School of Mathematics and Physics, \\ Queen's University Belfast, Belfast BT7 1NN, Northern Ireland, United Kingdom \\ Vladimir V. Rybkin, Marcella Iannuzzi, Jürg Hutter \\ Department of Chemistry, University of Zurich, Winterthurerstrasse 190, Zurich 8057, Switzerland \\ (*D.W: d.wilkins@qub.ac.uk) \\ (*J.L:jinggang.lan@epfl.ch) \\ (Dated: October 5, 2021)
}

\begin{abstract}
We report the static and dynamical properties of liquid water at second-order Møller-Plesset perturbation theory level (MP2) with classical and quantum dynamics simulations using a neural network potential. We examined the temperature dependent radial distribution function, diffusion and vibrational dynamics. MP2 theory predicts an over-structured liquid water at ambient conditions, which may be attributed to the incomplete basis set. The excellent agreement with experimental structural properties as well as the diffusion constant is observed at an elevated temperature of $340 \mathrm{~K}$.
\end{abstract}

\section{INTRODUCTION}

Water is both an extremely common and an extremely unusual liquid [1], with anomalous properties including a decrease in density upon freezing [2]. Despite the numerous efforts that have been made to understand the phase behavior of water, it remains a challenge to both experiment and theory. Understanding how the physical properties of water, such as its hydrogen bond network, diffusion coefficient and phase diagram, is relevant to many outstanding scientific problems, including nano-fluidics, which is important in desalination, [3] ion transportation, [4] protein folding,[5] and energy conversion[6]. Hence, water has received great attention in both experimental and theoretical studies across diverse scientific disciplines.

Molecular dynamics (MD) simulations can provide very useful insights into the atomic details of water. There is a long standing effort to simulate water using MD methods.[710] The driving forces for MD can be obtained from either empirical force-field or $a b$ initio calculations. Many water models have been proposed such as $\operatorname{TIP} n \mathrm{P}$ family[11-13], SPC $[14,15]$, MB-Pol[16]. It is common to use density functional theory (DFT) for ab initio molecular dynamics. However, despite the fact that the DFT formalism is exact for ground state properties in principle, in practice approximations must be made in the exchange-correlation energy. In principle, the accuracy increases according to the "Jacob's ladder" of electronic structure theory, with the local density approximation (LDA), generalized gradient approximations (GGAs), meta-GGAs, hybrid functionals, and beyond e.g. double hybrids, random phase approximation (RPA), and correlated wave function methods, such as second-order Møller-Plesset perturbation theory (MP2). A properly cho- sen exchange-correlation functional has been found to predict reasonable binding energy for the water dimer, trimer, larger clusters, and ice as well as the structure of liquid water when combined with MD. LDA is unacceptable as it overbinds the water dimer by nearly a factor of 2.[17] Unsurprisingly, LDA predicts overstructured liquid water and a very small diffusion constant.[18] GGA functionals perform only slightly better. DFT-based MD at the GGA level, without dispersion corrections, often requires a much higher temperature than $300 \mathrm{~K}$ to maintain water in the liquid state. For example, one of the most popular functionals, PBE, can reproduce experimental radial distribution functions (RDFs) and density at $\mathrm{T}=440$ $\mathrm{K}$ and $\mathrm{P}=0.3 \mathrm{GPa}[19]$. Even when the dispersion correction is added a higher temperature is still required to reproduce the these properties. Grimme's dispersion correction to PBE does not significantly improve the structure of water.[20] BLYP with this correction requires simulation at $360 \mathrm{~K}$ to reproduce the correct RDF.[21] Among the GGA functionals, the revPBE-D3 tends to perform best for RDF, density, and diffusion coefficient at ambient conditions.[17] However, the results are very sensitive to the particular choice of dispersion functional. The revPBE+DRSLL functional tends to overestimate the volume of ice VIII by $20 \%$. [17]

Recent developments in computational power and methodology have made it possible to climb higher rungs beyond the GGA ladder such as meta-GGA, hybrid, and beyond. The meta-GGA functional SCAN yields the correct ordering of densities between liquid water and ice, at the same time predicting quantitative agreement with experiments at an elevated temperature of $330 \mathrm{~K}$. The revPBE0-D3 hybrid functional is able to predict the correct experimental RDF at room temperature.[22] In addition, with tremendous computational cost one can reach the fifth rung of the ladder with methods such as RPA and MP2. The MP2 theory gives excellent pre- 
dictions of the density at room temperature, while the calculated radial distribution functions are in fair agreement with experimental data[23] that tend to be attributed to the stronger dispersion interaction. Previous studies suggest that "MP2 water" is denser and cooler at ambient conditions compared both with experiment and with "DFT water". [24]

In addition to the underlying electronic structure theory another issue is accurate account of the quantum nature of the nuclei.[25, 26] Neglect of nuclear quantum effects may become one of the largest sources of error. For instance, there are subtle differences in RDFs upon the inclusion of NQEs, especially those that involve hydrogen atoms.[25] "Classical" water is more basic than "quantum" water (i.e., water in nature) with a $\mathrm{pH}$ of 8.6, which is about a 30 -fold change in the ionization constant.[25] Similar effects have also been found at metal-water interfaces[27] and for electron transfer in aqueous solutions[28]. Hence, the inclusion of nuclear quantum effects is often necessary to achieve high accuracy in molecular dynamics simulations.

Path integral molecular dynamics (PIMD) is a method to incorporate quantum mechanics into molecular dynamics by mapping each quantum particle onto a classical representation[29] Despite that combining electronic structure theory with PI methods is exceedingly computationally expensive as each particle is represented by several classical replica, each requiring a separate on-the-fly electronic structure calculation. For this reason, high-level electronic structure calculations coupled with quantum nuclear dynamics have only recently been made possible.[22] Thanks to recent development of neural network potential (NNP), [30, 31] an extensive study using a high-quality functional beyond local DFT is feasible even when combined with path-integral molecular dynamics. For instance, the densities of water as predicted by neural network potential trained on revPBE0-D3 data agree with the experiment to within $3 \%$ for both liquid water and ice Ih and Ic.[32] Accurate and efficient quantum vibrational spectra of water can also be obtained for bulk and interfacial systems. [33, 34] Recently, quantum dynamics simulations with a driving force from a fifth-rung electronic structure leve have provided accurate determination of the structure, diffusion, and vibrational features of water and aqueous solvated electron .[35, 36]

\section{COMPUTATIONAL DETAILS}

All MD simulations are performed by using i-PI code[37, 38] interfaced with the LAMMPS [39] code, which implements the BPNN potential using N2P2 [40]. We used 128 water molecules at their experimental density to perform longtimescale molecular dynamics simulations. To achieve efficient canonical sampling while minimally perturbing the dynamics, the temperature of classical MD simulations was controlled using a stochastic velocity rescaling (SVR) thermostat [41], with a time constant of $1000 \mathrm{fs}$. A timestep of $0.5 \mathrm{fs}$ was used.

Path integral molecular dynamics (PIMD) [29] was used to incorporate nuclear quantum effects into the calculation of static properties. 64 ring-polymer beads were used, which is sufficient to converge the properties of interest even at low temperatures. For dynamical properties, thermostatted ring polymer molecular dynamics (TRPMD) [42], which has been shown to predict accurate vibrational dynamics of liquid water [22,43], and partially adiabatic centroid molecular dynamics (PACMD) [44]. Both methods predict similar diffusion constants but either may have deficiencies when computing vibrational spectra especially in stretching regions.[22, 45] A timestep of 0.25 fs was used for PIMD and TRPMD calculations, and 0.05 fs for PACMD. The temperature in PIMD calculations was controlled using a global path integral Langevin equation (PILE-G) thermostat,[46] with a time constant of $1000 \mathrm{fs}$.

Static and dynamical properties are calculated based on 200 ps trajectory at each temperature, other than PACMD, which used only $20 \mathrm{ps}$ for additional vibrational density of states (VDOS) calculations.

The dynamical properties of liquid water can be measured by diffusion coefficient which can be estimated by Einstein's relation:

$$
D=\frac{1}{6 t}\left\langle|r(t)-r(0)|^{2}\right\rangle
$$

The VDOS from classical MD or PIMD are calculated using the Fourier transform of the velocity autocorrelation,

$$
C_{v v}(\omega)=\int\langle v(\tau) v(t+\tau)\rangle_{\tau} e^{-i \omega t} d t,
$$

with the quantum autocorrelation function computed using the centroid velocity.

The starting point for our NN potential is that of Ref. 36, at the MP2 level. The reference data obtained at room temperature may be highly correlated, leading to incorrect dynamics at temperatures other than $300 \mathrm{~K}$. The potential was retrained by generating a large set of configurations of 64 molecules at temperatures from $240 \mathrm{~K}$ to $350 \mathrm{~K}$ using replica exchange molecular dynamics in the NPT ensemble. 2000 additional structures were selected used CUR selection based on their atomic fingerprints [47] and added to the training set. MP2 calculations [48, 49] with triple-zeta quality correlation-consistent basis sets [50] were carried out using CP2K [51, 52].NNP training was implemented in N2P2 [40].

\section{RESULTS}

\section{A. Structure of MP2 water at different temperatures}

To shed light on the structure of water at different temperatures, the radial distribution functions (RDFs) of oxygenoxygen, oxygen-hydrogen, and hydrogen-hydrogen pairs have been calculated, as shown in Fig.1 The first point to note here is that the RDFs as obtained from an NN potential at $300 \mathrm{~K}$ are in good agreement with the previous results from Ref.23 $g_{\mathrm{OO}}(\mathrm{r})$ tends to agree with experimental observations regardless of a much higher first maximum. In general, MP2 is more likely to overbind noncovalent complexes. 

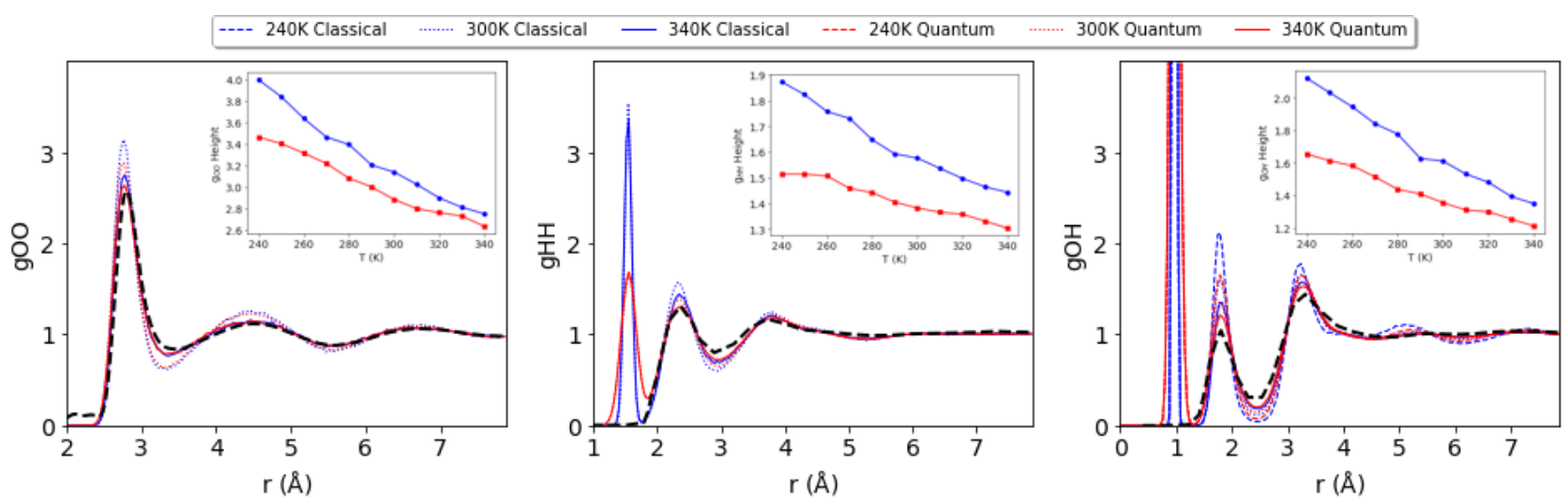

FIG. 1. Temperature dependence of radial distribution function as obtained from classical (solid line) and quantum (dashed line) simulations from 128 water models, in comparison to the experimental values at room temperature (black dashed line).
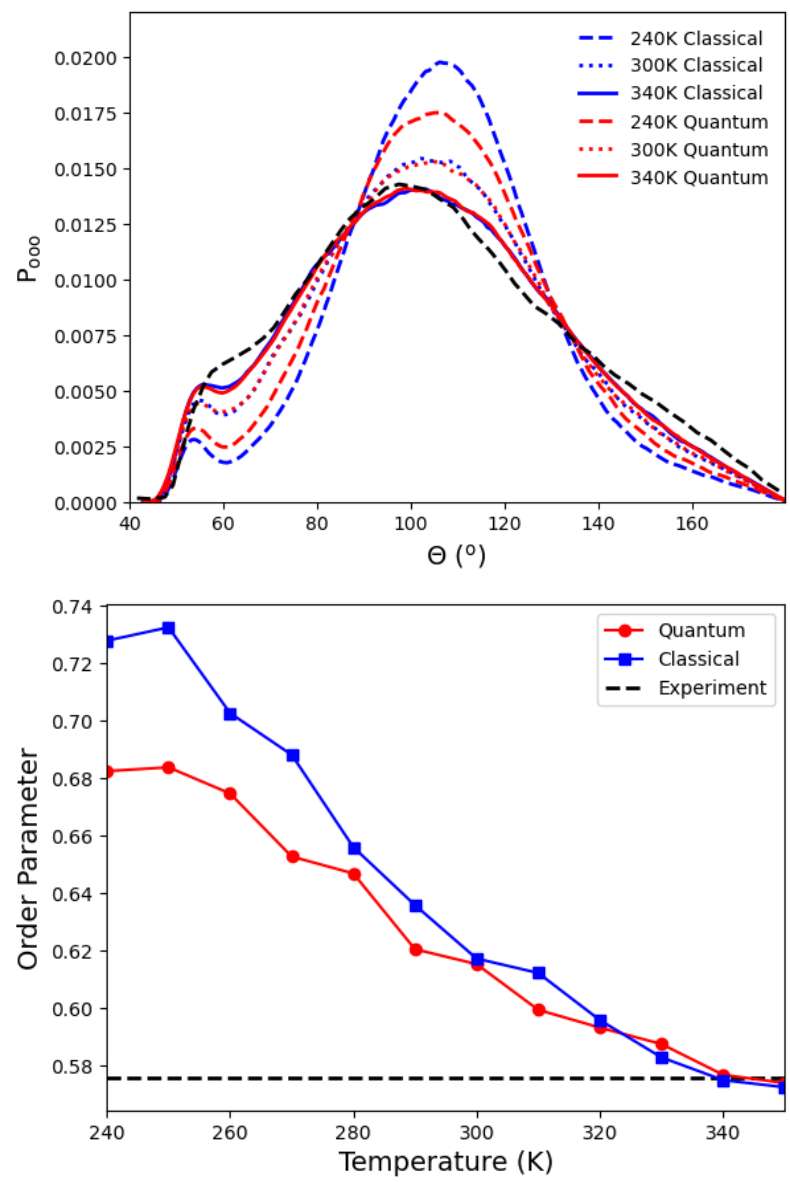

FIG. 2. The oxygen-oxygen-oxygen triplet angular distribution as obtained from classical and quantum dynamics at multiple temperatures. The resulting tetrahedral order parameter of liquid water is shown as a function of temperature. The experimental data at $298 \mathrm{~K}$ are marked in black dashed line as comparison.

The imperfect agreement between MP2 simulations and the experimental structure may be attributed to an insufficient ba- sis set. Calculations are carried out using a triple- $\zeta$ basis set. MP2 is known to overestimate dispersion when a relatively small basis set is applied.[53-56] Previous studies indicate that as the basis set increases from double- $\zeta$ to triple- $\zeta$ to quadruple- $\zeta$, the mean absolute error in the binding energies relative to the complete basis set (CBS) values decreases from 2.18 to 1.74 to $0.99 \mathrm{kcal} / \mathrm{mol}$ for the water clusters $(\mathrm{n}=2$ 10). The relatively small basis set overestimates the binding energies compared to the CBS.[56] The global minima of water clusters $(n=2-6)$ at the MP2 level agrees with the CCSD(T) level of theory.[57] However, MP2 systematically contracts the nearest-neighbor $\mathrm{O}-\mathrm{O}$ separation in water clusters by $0.005-0.022 \AA$ with the average of $0.016 \AA$. In this respect, MP2 tends to overestimate binding energies compared $\operatorname{CCSD}(\mathrm{T})$ results at the same quality of basis set (aug-ccpVDZ). [57] When the binding energies are extrapolated to the CBS limit, both MP2 and CCSD(T) predict very similar values. The differences between two methods are generally lower than $0.5 \mathrm{kcal} / \mathrm{mol}$.[57]

Given the fact MP2 with triple- $\zeta$ overbinds and overestimates the dispersion, it is not surprising that MP2 water at room temperature over-structures and behaves like ice. The temperature dependence of the RDFs as shown in Fig.1 from 240 to $350 \mathrm{~K}$ was also examined. The RDFs are strongly affected by the temperature with a negative correlation at each peak. Temperature effects are more pronounced at the first peaks of $g_{\mathrm{OO}}(r)$ which concerns the structure of the first solvation shell. As the temperature increases, the water becomes less ordered with a relatively lower intensity of $g_{\mathrm{OO}}(r)$ at the first peak. NQEs broaden and weaken the intensity of the first peaks in $g_{\mathrm{OO}}(r)$ and these effects are more pronounced at a lower temperature. A temperature difference of $20-30 \mathrm{~K}$ in classical simulations is required to give the same height of $g_{\mathrm{OO}}(r)$ as in quantum simulations, indicating that the quantum simulation is hotter.[9, 58] Indeed, classical MD simulations of water are often carried out at an elevated temperature to mimic the quantum effect of oxygen and yield an improved description of the local structure of water.[9] In fact, NQEs are more significant when light atoms are involved. Such simulation protocol with an artificial elevation of $30 \mathrm{~K}$ may 
fail dramatically in describing the $g_{\mathrm{HH}}(r)$ and $g_{\mathrm{OH}}(r)$.[9] As shown in Fig.1(b), NQEs significantly broaden the first and second peaks of $g_{\mathrm{HH}}(r)$ and $g_{\mathrm{OH}}(r)$. Similarly, we also plot the temperature dependence of the height of the second peaks of $g_{\mathrm{HH}}(r)$ and $g_{\mathrm{OH}}(r)$. It is evident that the temperature difference may be more than $70 \mathrm{~K}$ for $g_{\mathrm{HH}}(r)$ and $50 \mathrm{~K}$ for $g_{\mathrm{OH}}$ at the second peak of the RDFs. More importantly, the broadening of the first peaks of $g_{\mathrm{HH}}(r)$ and $g_{\mathrm{OH}}(r)$ cannot be reproduced with an elevated temperature even up to $70 \mathrm{~K}$. Classical MD simulations only sample within the range of the thermal energy $k T$, which is far below the zero-point energy of an $\mathrm{O}-\mathrm{H}$ stretch. Accurate RDFs can be achieved from MP2 theory with a higher temperature of $340 \mathrm{~K}$, as shown by our classical and quantum dynamics. As in Fig.1, the experimental RDFs at room temperature are given in the dashed black lines. The calculated $g_{\mathrm{OO}}(r), g_{\mathrm{HH}}(r)$ and $g_{\mathrm{OH}}(r)$ match perfectly with the experimental value at $340 \mathrm{~K}$. To further understand the local structure of the water molecules, we further calculate the distribution oxygen-oxygen-oxygen triplet angles within the first solvation shell and the tetrahedral order parameter $p$, defined as

$$
q=1-\frac{3}{8} \sum_{i=1}^{3} \sum_{j=i+1}^{4}\left(\cos \left(\theta_{i j}+\frac{1}{3}\right)^{2}\right.
$$

where $\theta_{i j}$ is the angle formed by the central water molecules and its two neighboring water molecules $i$ and $j$. To calculate the distribution of oxygen-oxygen-oxygen triplet angles within the first solvation shell, we take the cutoff distance of two oxygen atoms of $3.35 \AA$, which yields an average coordination number of 4 . An order parameter of $q=1$ defines a perfect tetrahedral local environment, and $q$ decreases as the structure of water become less ordered and tetrahedral. As shown in Fig.2, the experimental triplet angles show a weak shoulder at around $60^{\circ}$ and a broad strong peak at around $100^{\circ}$. We present the calculated $\mathrm{P}_{\text {ooo }}$ at three different temperatures $-240 \mathrm{~K}, 300 \mathrm{~K}$, and $340 \mathrm{~K}$. At the lower temperature of $240 \mathrm{~K}$, NQEs strongly adjust the local environment of water, resulting in a less ordered water structure. The order parameter as estimated from quantum simulations is about 0.68 which is lower than that of 0.72 from classical simulations. The difference of local parameters becomes less with increasing the temperature. At room temperature, MP2 predicts a local parameter of 6.15 for quantum simulations (6.17 for classical), which is higher than the prediction from the fragmentbased MP2/aug-cc-pVDZ simulation.[59] The inconsistency may come from the choice of basis sets and the employment of fragmentation methods. Beyond room temperature, the local parameter from classical and quantum simulations is almost identical (see Fig.2). The local order parameter at $340 \mathrm{~K}$ $\left(q_{\text {quantum }}=0.577, q_{\text {classical }}=0.575\right)$ predict almost exact number compared to the experimental value of 0.570 . The calculated triplet angles distributions predict an accurate line shape at around $100^{\circ}$, but a weaker shoulder at $56^{\circ}$ instead of $60^{\circ}$.

\section{B. Dynamical properties}

It is difficult to converge the diffusion coefficient using $a b$ initio molecular dynamics due to its high computational cost, especially when combined with high-level electronic structure theory beyond standard GGA. Diffusion coefficients from high-level electronic structure theory have been only made available by using a multiple time stepping (MTS) method. For instance, Marsalek and Markland carried out revPBE0-D3 calculations using ring-polymer contraction with MTS methods. The calculated diffusion coefficient of liquid water at 300 $\mathrm{K}$ is determined to be 2.67 and $2.29 \times 10^{-6} \mathrm{~cm}^{2} / \mathrm{s}$ from classical and quantum simulations respectively. The inclusion of NQEs decreases the diffusion coefficient by 30\%. [22] Del Ben and co-workers combined a hybrid functional (PBEW1D3) with MP2, using fast (0.25 fs) and slow (2.5 fs) time steps to calculate the dynamical property of MP2 water. Their analysis is based on two trajectories of each roughly $10 \mathrm{ps}$ as obtained in the NVE ensemble, and the self-diffusion constant values obtained are 0.67 and $0.77 \times 10^{-6} \mathrm{~cm}^{2} / \mathrm{s}$ at 300 and $307 \mathrm{~K}$, respectively. At room temperature, our simulations predict a diffusion constant of 0.566 (0.693) and 0.609 $(0.737) \times 10^{-6} \mathrm{~cm}^{2} / \mathrm{s}$ from classical and quantum dynamics (the quantities in brackets are after finite size correction[60]), which is in fair agreement with previous simulations[61]. In fact, a classical MD simulation with a limited timescale of 1020 ps may yield statistical error bars of more than $20 \%$ on dynamical quantities such as the diffusion coefficient. That may explain the minor inconsistency between our values and previous results.[22, 61] Nevertheless, these values are below the experimental diffusion at room temperature, and more similar to a lower-temperature diffusion. In Fig.3, we plotted the temperature-dependent diffusion coefficient as obtained from classical and quantum dynamics. The diffusion constants are systematically underestimated by MP2 theory for both classical and quantum simulations. When the experimental diffusion constant is shifted to a higher temperature by $40 \mathrm{~K}$, the diffusion constant as calculated from our simulations matches with experimental values. Previous studies show that NQEs contribute a $30 \%$ decrease to the self-diffusion of liquid water using revPBE0-D3 functional and a similar slowdown has also been observed using revPBE-D3 functional.[22] Unlike the revPBE0-D3 functional, the diffusion of water upon including NQEs are enhanced by $10-70 \%$ from 240 to $320 \mathrm{~K}$, while a slow down of diffusion by $3-7 \%$ have been observed beyond $330 \mathrm{~K}$.

To understand the vibrational dynamics of MP2 water, we calculate the density of states (VDOS) from the Fourier Transform of the velocity-velocity auto-correlation function at different temperatures. As shown in Fig.4, classical MD simulations overestimate the $\mathrm{OH}$-stretch mode and bend mode by around $300 \mathrm{~cm}^{-1}$ and $40^{-1}$ compared to experiment at room temperature (with the experimental $\mathrm{OH}$ stretch at $3380 \mathrm{~cm}$ ). In quantum simulations, the $\mathrm{O}-\mathrm{H}$ stretching frequency is redshifted from $3685 \mathrm{~cm}^{-1}$ to $3524 \mathrm{~cm}^{-1}$ (from TRPMD) and $3442 \mathrm{~cm}^{-1}$ (PACMD), with the latter agreeing better with experiment. [63]

NQEs also contribute to the improvement of the bending 


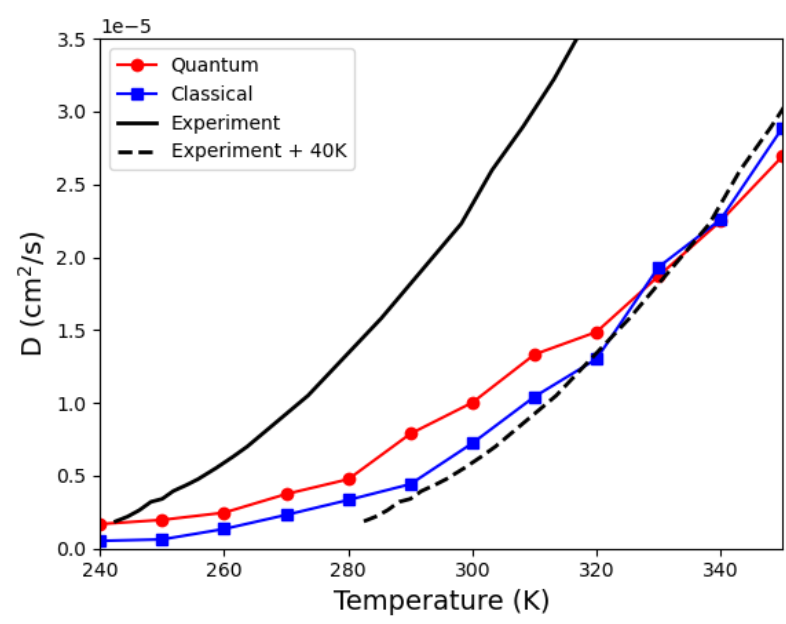

FIG. 3. Temperature dependence of the diffusion coefficient as obtained from classical and quantum simulations, in comparison to the experimental values (black line)[62] and its shifted value by $40 \mathrm{~K}$.

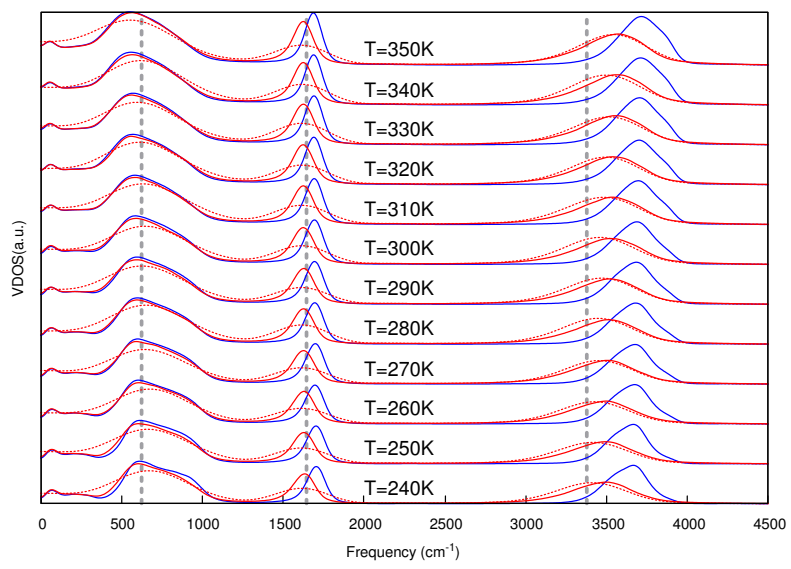

FIG. 4. Temperature dependent vibrational density of states from 240 $\mathrm{K}$ to $350 \mathrm{~K}$. The blue lines are obtained from classical simulations, while the red lines are from TRPMD, the red dashed-lines are from PACMD simulations. Vertical lines show experimental vibrational frequencies.

mode (experimentally $1637 \mathrm{~cm}^{-1}$ in the IR measurement) from 1693 (classical) to 1624 (quantum). While the agreement of MP2 calculations with experiments is very good, that of revPBE0-D3 water is somewhat better, particularly for the $\mathrm{O}-\mathrm{H}$ stretching peak [26]. The PACMD results for the librational and bending modes in water are reasonably good across the range of temperatures studied, but the agreement between the experimental and PACMD O-H stretching modes improves as temperature is decreased, with quantum results at
$240 \mathrm{~K}$ giving the best agreement with experiments.[63]

\section{CONCLUSIONS}

In this work, we simulated MP2 water using a neural network potential at different temperatures using classical and quantum dynamics. MP2 theory, though belonging to the fifth rung of the electronic-structure "Jacob's ladder", is unable to predict accurately the static and dynamical properties of liquid water. This may be because an insuffiently large basis set causes MP2 theory to overestimate the Van der Waals interaction. This may be improved upon by increasing the basis set to quadruple- $\zeta$ and larger, or by estimating the difference between the results of small and large basis sets using cluster models and decomposing the error into two- and threebody interaction terms, which can be used to further cancel the basis-set error.

Nuclear quantum effects reduce the first peak of the $g_{\mathrm{OO}}(r)$ radial distribution function at room temperature but do not perfectly match with the experimental RDF.[61] However, an excellent agreement with experimental RDFs is observed at an elevated temperature of $340 \mathrm{~K}$, at which MP2 predicts excellent static properties of liquid water compared to the experimental data, as characterized by the RDFs, triplet angles distribution, and tetrahedral local order parameters. It is possible that the elevated temperature acts to cancel the error due to an insufficient basis set, with higher kinetic energy compensating for the overestimation of dispersion by MP2 theory. Although our NNP accurately captures a range of static and dynamical properties, many properties of water such as the dielectric constant and vibrational spectra are not yet available. Future work will focus on the modelling of these properties using symmetry-adapted machine learning methods [64].

\section{ACKOWLEDGEMENTS}

This work is funded by the Swiss National Science Foundation (SNSF) Sinergia grant and the University Research Priority Program (URPP) for solar light to chemical energy conversion (LightChEC) of the University of Zurich. This work was also supported by a grant from the Swiss National Supercomputing Centre (CSCS) under Project ID uzh1, s1043. VRR has been supported by the SNSF in the form of Ambizione grant No. PZ00P2_174227.

\section{DATA AVAILABILITY}

Data generated and analyzed for this study that are not included in this article are available at https://www.materialscloud.org.
[1] L. G. M. Pettersson, R. H. Henchman, and A. Nilsson, Chemical reviews 116, 7459 (2016).
[2] P. Gallo, K. Amann-Winkel, C. A. Angell, M. A. Anisimov, F. Caupin, C. Chakravarty, E. Lascaris, T. Loerting, A. Z. 
Panagiotopoulos, J. Russo, et al., Chemical reviews 116, 7463 (2016).

[3] C. Herrero, G. Tocci, S. Merabia, and L. Joly, Nanoscale 12, 20396 (2020).

[4] L. Joly, R. H. Meißner, M. Iannuzzi, and G. Tocci, ACS Nano (2021), 10.1021/acsnano.1c05931, pMID: 34491721, https://doi.org/10.1021/acsnano.1c05931.

[5] V. Daggett, Chemical reviews 106, 1898 (2006).

[6] M. G. Walter, E. L. Warren, J. R. McKone, S. W. Boettcher, Q. Mi, E. A. Santori, and N. S. Lewis, Chemical reviews 110, 6446 (2010).

[7] G. A. Cisneros, K. T. Wikfeldt, L. Ojamäe, J. Lu, Y. Xu, H. Torabifard, A. P. Bartók, G. Csányi, V. Molinero, and F. Paesani, Chemical reviews 116, 7501 (2016).

[8] A. Rahman and F. H. Stillinger, The Journal of Chemical Physics 55, 3336 (1971).

[9] J. A. Morrone and R. Car, Physical review letters 101, 017801 (2008).

[10] K. Laasonen, M. Sprik, M. Parrinello, and R. Car, The Journal of chemical physics 99, 9080 (1993).

[11] R. W. Hockney and J. W. Eastwood, Computer simulation using particles (crc Press, 2021).

[12] M. W. Mahoney and W. L. Jorgensen, The Journal of Chemical Physics 114, 363 (2001).

[13] H. W. Horn, W. C. Swope, J. W. Pitera, J. D. Madura, T. J. Dick, G. L. Hura, and T. Head-Gordon, The Journal of chemical physics 120, 9665 (2004).

[14] H. Berendsen, J. Grigera, and T. Straatsma, Journal of Physical Chemistry 91, 6269 (1987).

[15] H. J. Berendsen, J. P. Postma, W. F. van Gunsteren, and J. Hermans, in Intermolecular forces (Springer, 1981) pp. 331-342.

[16] V. Babin, G. R. Medders, and F. Paesani, Journal of chemical theory and computation 10, 1599 (2014).

[17] M. J. Gillan, D. Alfe, and A. Michaelides, The Journal of chemical physics 144, 130901 (2016).

[18] E. Liberatore, R. Meli, and U. Rothlisberger, Journal of chemical theory and computation 14, 2834 (2018).

[19] S. Yoo, X. C. Zeng, and S. S. Xantheas, The Journal of chemical physics 130, 221102 (2009).

[20] K. Forster-Tonigold and A. Groß, The Journal of chemical physics 141, 064501 (2014).

[21] S. Yoo and S. S. Xantheas, The Journal of chemical physics 134, 121105 (2011).

[22] O. Marsalek and T. E. Markland, The journal of physical chemistry letters 8, 1545 (2017).

[23] M. Del Ben, M. Schönherr, J. Hutter, and J. VandeVondele, The journal of physical chemistry letters 4, 3753 (2013).

[24] S. Y. Willow, X. C. Zeng, S. S. Xantheas, K. S. Kim, and S. Hirata, The journal of physical chemistry letters 7, 680 (2016).

[25] M. Ceriotti, W. Fang, P. G. Kusalik, R. H. McKenzie, A. Michaelides, M. A. Morales, and T. E. Markland, Chemical reviews 116, 7529 (2016).

[26] T. E. Markland and M. Ceriotti, Nature Reviews Chemistry 2, 1 (2018).

[27] J. Lan, V. V. Rybkin, and M. Iannuzzi, The journal of physical chemistry letters 11, 3724 (2020).

[28] V. V. Rybkin and J. VandeVondele, The Journal of Physical Chemistry Letters 8, 1424 (2017), pMID: 28296416, https://doi.org/10.1021/acs.jpclett.7b00386.

[29] M. Parrinello and A. Rahman, The Journal of chemical physics 80, 860 (1984).

[30] J. Behler and M. Parrinello, Physical Review Letters 98, 146401 (2007).
[31] L. Zhang, J. Han, H. Wang, R. Car, and E. Weinan, Physical review letters 120, 143001 (2018).

[32] B. Cheng, E. A. Engel, J. Behler, C. Dellago, and M. Ceriotti, Proceedings of the National Academy of Sciences 116, 1110 (2019).

[33] S. Shepherd, J. Lan, D. M. Wilkins, and V. Kapil, arXiv preprint arXiv:2108.03056 (2021).

[34] V. Kapil, D. M. Wilkins, J. Lan, and M. Ceriotti, The Journal of chemical physics 152, 124104 (2020).

[35] Y. Yao and Y. Kanai, The journal of physical chemistry letters 12, 6354 (2021).

[36] J. Lan, V. Kapil, P. Gasparotto, M. Ceriotti, M. Iannuzzi, and V. V. Rybkin, Nature communications 12, 1 (2021).

[37] V. Kapil, M. Rossi, O. Marsalek, R. Petraglia, Y. Litman, T. Spura, B. Cheng, A. Cuzzocrea, R. H. Meißner, D. M. Wilkins, et al., Computer Physics Communications 236, 214 (2019).

[38] M. Ceriotti, J. More, and D. E. Manolopoulos, Computer Physics Communications 185, 1019 (2014).

[39] S. Plimpton, Journal of computational physics 117, 1 (1995).

[40] A. Singraber, J. Behler, and C. Dellago, Journal of Chemical Theory and Computation 15, 1827 (2019).

[41] G. Bussi, D. Donadio, and M. Parrinello, The Journal of Chemical Physics 126, 014101 (2007).

[42] M. Rossi, M. Ceriotti, and D. E. Manolopoulos, The Journal of Chemical Physics 140, 234116 (2014).

[43] R. L. Benson, G. Trenins, and S. C. Althorpe, Faraday Discussions 221, 350 (2019).

[44] T. D. Hone, P. J. Rossky, and G. A. Voth, J. Chem. Phys. 124, 154103 (2006).

[45] S. Habershon, G. S. Fanourgakis, and D. E. Manolopoulos, The Journal of chemical physics 129, 074501 (2008).

[46] M. Ceriotti, M. Parrinello, T. E. Markland, and D. E. Manolopoulos, The Journal of chemical physics 133, 124104 (2010).

[47] G. Imbalzano, A. Anelli, D. Giofré, S. Klees, J. Behler, and M. Ceriotti, The Journal of Chemical Physics 148, 241730 (2018).

[48] M. Del Ben, J. Hutter, and J. VandeVondele, The Journal of Chemical Physics 143, 102803 (2015).

[49] V. V. Rybkin and J. VandeVondele, Journal of Chemical Theory and Computation 12, 2214 (2016).

[50] M. Del Ben, J. Hutter, and J. VandeVondele, Journal of Chemical Theory and Computation 9, 2654 (2013).

[51] J. Hutter, M. Iannuzzi, F. Schiffmann, and J. VandeVondele, Wiley Interdisciplinary Reviews: Computational Molecular Science 4, 15 (2014).

[52] T. D. Kühne, M. Iannuzzi, M. Del Ben, V. V. Rybkin, P. Seewald, F. Stein, T. Laino, R. Z. Khaliullin, O. Schütt, F. Schiffmann, D. Golze, J. Wilhelm, S. Chulkov, M. H. Bani-Hashemian, V. Weber, U. Borštnik, M. Taillefumier, A. S. Jakobovits, A. Lazzaro, H. Pabst, T. Müller, R. Schade, M. Guidon, S. Andermatt, N. Holmberg, G. K. Schenter, A. Hehn, A. Bussy, F. Belleflamme, G. Tabacchi, A. Glöß, M. Lass, I. Bethune, C. J. Mundy, C. Plessl, M. Watkins, J. VandeVondele, M. Krack, and J. Hutter, The Journal of Chemical Physics 152, 194103 (2020).

[53] J. Kim and K. S. Kim, The Journal of chemical physics 109, 5886 (1998).

[54] S. S. Xantheas, C. J. Burnham, and R. J. Harrison, The Journal of chemical physics 116, 1493 (2002).

[55] S. S. Xantheas and E. Aprà, The Journal of chemical physics 120, 823 (2004). 
[56] B. Temelso, K. A. Archer, and G. C. Shields, The Journal of Physical Chemistry A 115, 12034 (2011).

[57] E. Miliordos, E. Aprà, and S. S. Xantheas, The Journal of chemical physics 139, 114302 (2013).

[58] M. Chen, H.-Y. Ko, R. C. Remsing, M. F. C. Andrade, B. Santra, Z. Sun, A. Selloni, R. Car, M. L. Klein, J. P. Perdew, et al., Proceedings of the National Academy of Sciences 114, 10846 (2017).

[59] J. Liu, X. He, J. Z. Zhang, and L.-W. Qi, Chemical science 9, 2065 (2018).

[60] I.-C. Yeh and G. Hummer, The Journal of Physical Chemistry B 108, 15873 (2004).

[61] M. Del Ben, J. Hutter, and J. VandeVondele, The Journal of chemical physics 143, 054506 (2015).
[62] P. Tofts, D. Lloyd, C. Clark, G. Barker, G. Parker, P. McConville, C. Baldock, and J. Pope, Magnetic Resonance in Medicine: An Official Journal of the International Society for Magnetic Resonance in Medicine 43, 368 (2000).

[63] M. W. Chase Jr, J. Phys. Chem. Ref. Data, Monograph 9 (1998).

[64] A. Grisafi, D. M. Wilkins, G. Csányi, and M. Ceriotti, Phys. Rev. Lett. 120, 036002 (2018).

[65] J. VandeVondele and J. Hutter, The Journal of Chemical Physics 127, 114105 (2007).

[66] I. R. Craig and D. E. Manolopoulos, The Journal of Chemical Physics 121, 3368 (2004). 